\title{
Neutron Diffraction Measurements of Residual Stress and Mechanical Testing of Pressure Sintered Metal-Ceramic Composite Systems
}

\author{
Karl Toppler ${ }^{1, a^{*}}$, Vladimir Luzin ${ }^{1, b}$, Michael Saleh ${ }^{1, c}$, Andrew Ruys ${ }^{2, d}$, \\ $\mathrm{Kaveh} \mathrm{Kabir}^{3, \mathrm{e}}$ and Dorji Chavara ${ }^{1, \mathrm{f}}$ \\ ${ }^{1}$ Australian Nuclear Science and Technology Organisation. Locked Bag 2001, Kirrawee DC, \\ NSW, 2232. Australia \\ ${ }^{2}$ School of Aerospace, Mechanical and Mechatronic Engineering, University of Sydney. NSW \\ 2006. Australia \\ ${ }^{3}$ Dept. of Engineering, Macquarie University, NSW.2109. Australia

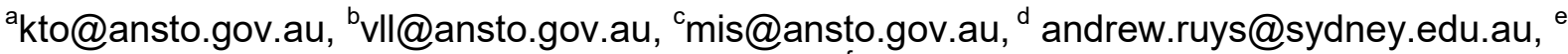

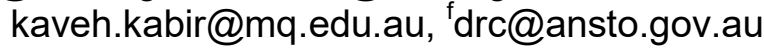

Keywords: Functionally Graded Materials, FGM, Residual Stress, Metal-Ceramic Composite

\begin{abstract}
Functionally graded materials (FGMs) are composite materials which vary in phase composition, microstructure and properties over one or more dimensions. They are a good potential choice for nuclear reactor components as they can be engineered to effectively resist corrosion and radiation damage. In the case of a metal-ceramic FGM, they can mate the strength and ductility of a metal with the hardness and toughness of a ceramic. A series of composite samples of variable metal-ceramic ratios was manufactured by hot uniaxial pressing in cylinders. Bulk uniform samples of a certain composition were manufactured as a more efficient way of studying FGMs without the extreme gradient required in practical applications. Thermally and mechanically generated stresses, inherent in composites, frequently create conditions for micro-cracking development, depending on the material's micro-structural characteristics and the thermomechanical processing route. Bulk stress measurements in the prepared samples were carried out on the Kowari diffractometer on the OPAL reactor at ANSTO. Both phases - metal matrix and ceramic inclusions - were measured in both axial and lateral directions for full characterisation of the composite stress state. When compared against analytical evaluation, experimental results, for some samples, demonstrated significant stress relaxation with micro-cracking being the main suspect.
\end{abstract}

\section{Introduction}

Functionally graded materials (FGMs) were first proposed as an advanced engineering material in 1972, and have been actively pursued since then. They are proposed in areas such as biomaterials, aerospace, chemical plants, mining and building material commodities[1, 2]. Within the nuclear industry FGMs can be engineered to effectively resist corrosion, radiation and be used as potential nuclear reactor components; first wall for fusion reactors and fuel pellets. They have been proposed as potential plasma facing components (PFCs) in which the ideal PFC would gradually vary from a refractory material (e.g. tungsten, plasma face) to a heat sink material (e.g. copper). In the case of a metal-ceramic FGM, the composite can be engineered to mate the strength and ductility of a metal with the hardness and toughness of a ceramic [3-5]. FGMs have been successfully fabricated using various methods to produced thin film/interfacial FGMs or bulk FGMs (layered/continuous) [3]. 
The FGM manufacturing routes can be broadly classified as layered or continuous based on their gradient profile. As the name suggests, layered FGMs have distinct step changes of material composition/properties across the layered axis, in contrast to continuous gradient FGMs, which do not [3].

Being a composite material, FGMs have all the attributes of composites; with inhomogeneous microstructure, material properties, etc., while characterisation of their mechanical state requires description in terms of micromechanics. Experimental studies of FGMs are difficult due to the gradients of properties. A more economical way of doing this is through the preparation and characterisation of bulk samples of uniform and pre-determined composition and properties, suitable for mechanical testing as well as for neutron diffraction studies of the micro-stress state. This study examines the micro-mechanical behaviour of a series of composite samples of

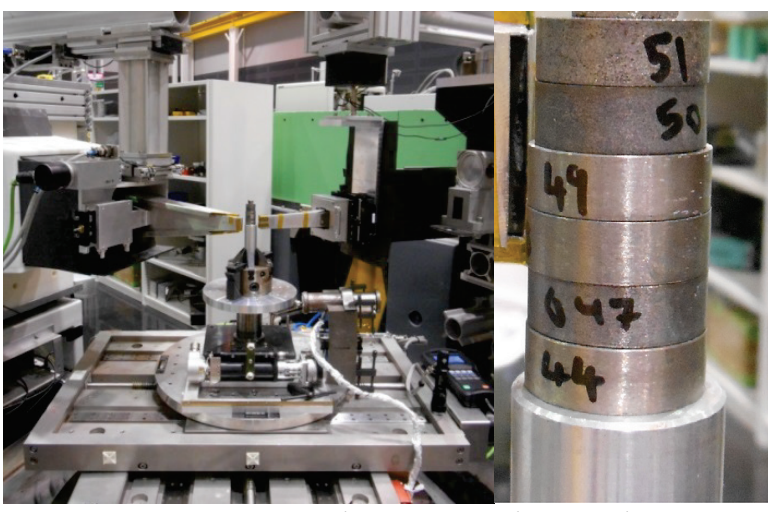

Fig. 1. Experimental setup and samples on the KOWARI diffractometer. variable composition, to predict the likely properties of a FGM.

\section{Sample synthesis and characterisation}

The system analysed in the current study is a Molybdenum-Yttrium Oxide system. The pertinent material properties are shown in Table 1 and the as-received powder morphology was imaged using a scanning electron microscope. The samples were manufactured using a traditional powder processing route. To produce the required volume based composite analogues, the required mass of powder for each portion (i.e. ceramic or metallic) was calculated using theoretical densities (Table 1). Both portions were mixed with a tubular mixer for $10 \mathrm{~min}$. With the aid of a uniaxial press the mixed powder was pressed with a tool-steel die to $50 \mathrm{MPa}$. These pellets were hot pressed at $1600^{\circ} \mathrm{C}$ at a pressure of $40 \mathrm{MPa}$ for $1 \mathrm{~h}$.

Sample densities were measured as per AS1774.5:2014 [6] and compared to theoretical densities as a function of sample composition.

Light microscopy images were taken of these analogues to understand the fabricated macrostructure (Figure 3b). The elastic moduli were measured through the impulse exciation technique according to standards [7-9] using a GrindoSonic MK4 appartus. The Young's modulus for a rectangular bar sample is determined from the natural frequency corresponding to the first flexural mode of vibration.

Table 1. Material properties of the Mo-Y2O3 powders [3, 10]

\begin{tabular}{|l|c|c|}
\hline & Molybdenum $(\mathrm{Mo})$ & Yttrium Oxide $\left(\mathrm{Y}_{2} \mathrm{O}_{3}\right)$ \\
\hline Thermal Expansion $(\mathrm{CTE}),\left[\times 10^{-6} \mathrm{~K}^{-1}\right]$ & $4.8-5.1$ & 8.1 \\
\hline Density, $[\mathrm{g} / \mathrm{cc}]$ & 10.2 & 5.03 \\
\hline Melting Point, $\left[{ }^{\circ} \mathrm{C}\right]$ & 2610 & 2410 \\
\hline Crystal Structure & body centered cubic & cubic \\
\hline
\end{tabular}

To assess residual stress, both phases of the mixed composition samples were measured. Pure material samples were used as the $\mathrm{d}_{0}$ reference, as all samples were synthesised through the same thermo-mechanical treatment (i.e., pressure and temperature characteristics of the sintering process). A wavelength of $\lambda=1.67 \AA$ was used to measure the $\mathrm{Mo}(211)$ and $\mathrm{Y}_{2} \mathrm{O}_{3}(440)$ reflections at scattering angles of $81^{\circ}$ and $53^{\circ}$, respectively. The samples were stacked in series and measured while spinning continuously to achieve better statistics, as shown in Figure 1. 
For the cylindrical samples of diameter $25 \mathrm{~mm}$ and height $8-10 \mathrm{~mm}$, a relatively large gauge volume of $5 \times 5 \times 5 \mathrm{~mm}^{3}$ was used; it was assumed there was no macro-stress (samples were uniform and isotropic). However, the samples were measured in two principal directions (axial and lateral). Strains were measured with an accuracy of 5E10-5 in order to get an accuracy of $\sim 20 \mathrm{MPa}$ in the stress scale for metals, which was somewhat larger for the ceramics. In order to achieve this, the measurement time was adjusted according to the volume fraction and material, varying from 1 to 30 $\min$.

\section{Results and Discussion}

The metallic powder used to fabricate the FGMs had a significantly larger particle size than its ceramic counterpart (Figure 2). Ceramic particles were flaky and had a fractured appearance in contrast to the more cleaved spherical morphology of the metallic powders.
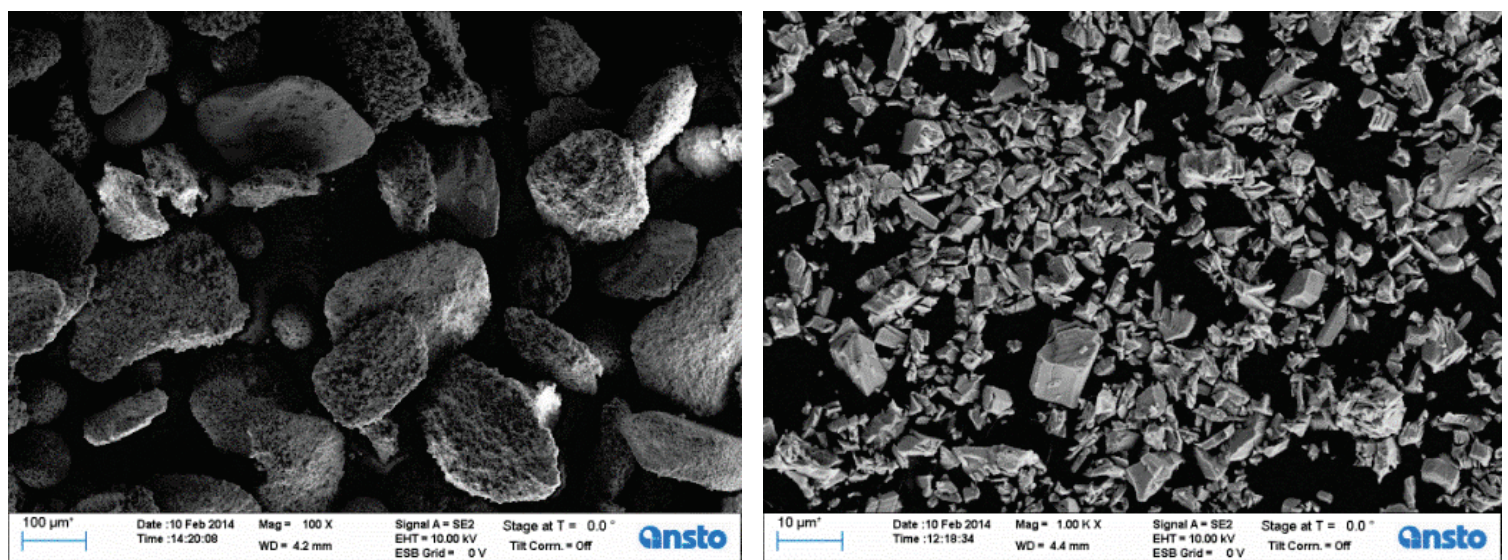

Fig. 2. Powder morphology for (a) Mo and (b) $\mathrm{Y}_{2} \mathrm{O}_{3}$ powders.
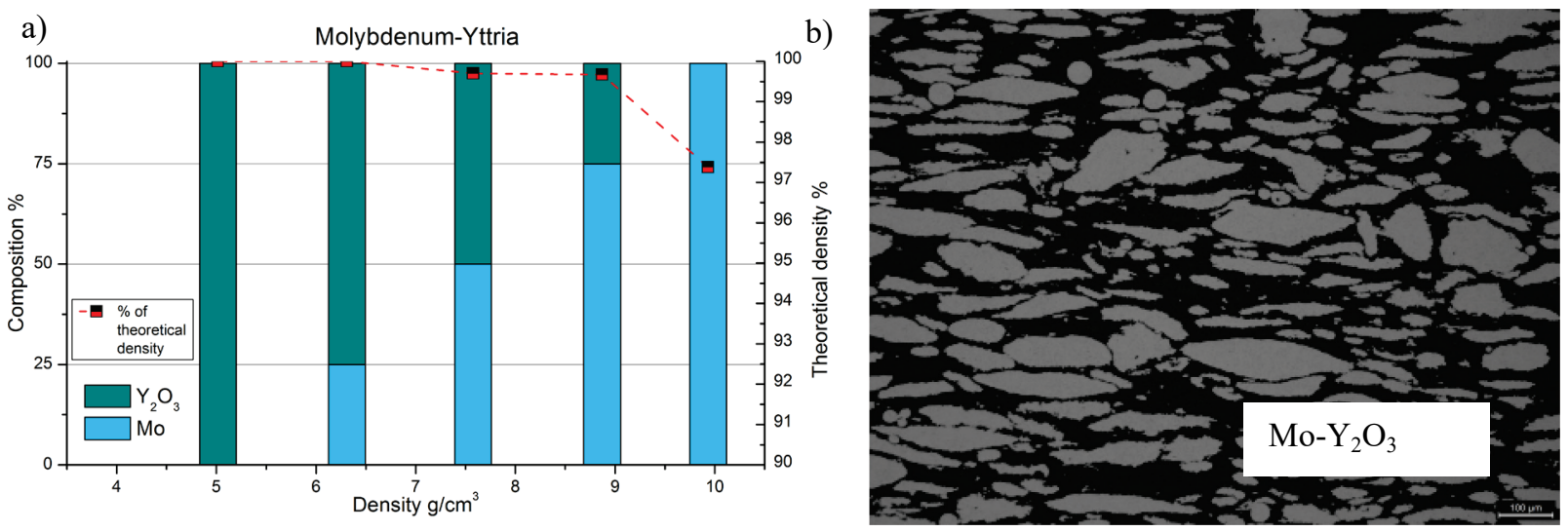

Fig. 3. (a) Density measurements of samples as function of the composition (b) Light microscopy micrograph with 50\% Mo (light grey phase) and 50\% $\mathrm{Y}_{2} \mathrm{O}_{3}$ (dark phase).

I he results of neutron residual stress analysis on the composite samples are shown in rigure 5 . I he overall trend of compressive stress in the Mo, with $\mathrm{Y}_{2} \mathrm{O}_{3}$ being in tension, suggests the stresses stem from the thermal mismatch [12]. However, the stress state is anisotropic with distinctly different axial and lateral components. Although the lateral component Mo compressive stress seems to be over evaluated (especially for the $25 \%$ sample), the stress balance, $f \cdot \sigma_{i}^{Y t t}+(1-f) \cdot \sigma_{i}^{M o}$, shows it is within experimental uncertainties for all components. 
Interphase micro-stresses explain the results, as no macro-stresses were detected in the composite samples, which were uniform, but not isotropic. Considering the manufacturing route (hightemperature uniaxial pressing), material properties of the different phases (coefficient of thermal expansion (CTE) and Young's Modulus (E) mismatch) and the microstructure details (anisotropic elongation with micro-cracking), a qualitative interpretation of the stress state in the composite was possible, as follows:

(i) The thermal stresses due to the mismatch in CTE were largely isotropic/hydrostatic, however, the oriented micro-cracking suggested a deviatoric stress component. The samples exhibited compressive stresses in Mo and tensile stresses in $\mathrm{Y}_{2} \mathrm{O}_{3}$ attributable to the discrepancy in the CTE ( 5.0 for Mo vs. 8.1 for $\mathrm{Y}_{2} \mathrm{O}_{3}$ ). The hydrostatic stress can be evaluated for the quasi-isotropic approximation using the HashinShtrikman relation [11] shown in Figure. 6. When compared with the experimental data, stress relaxation is observable for the samples with $f\left(Y_{2} \mathrm{O}_{3}\right)=0.5$ and
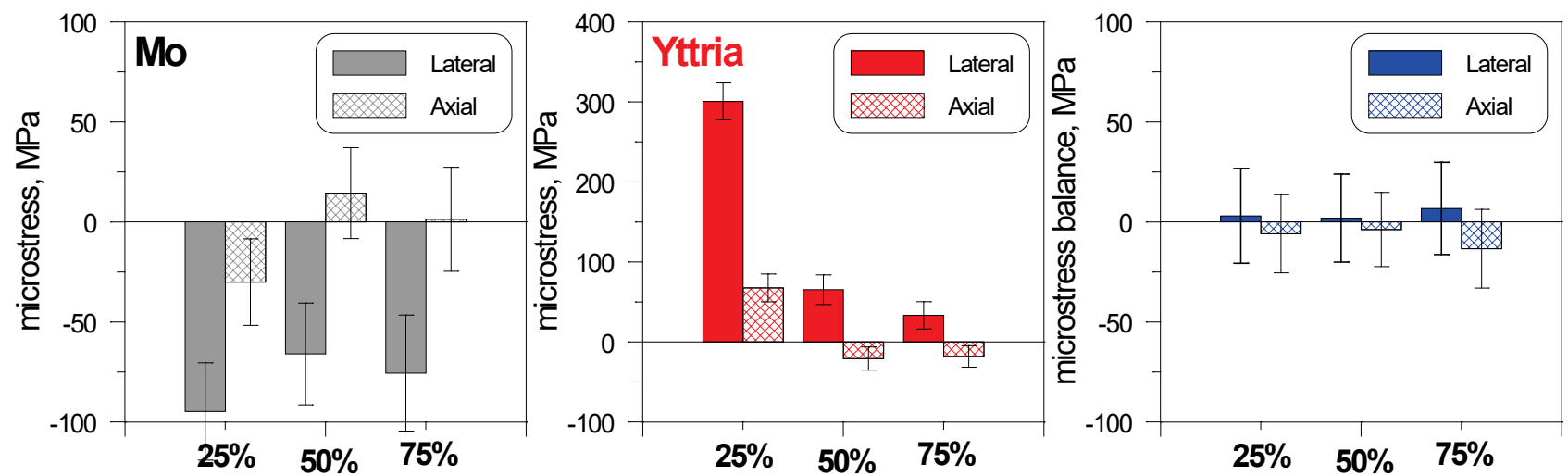

Fig. 5. a) Micro-stress distribution in the lateral and axial directions as functions of ceramic composition for the metal and ceramic phases. b) micro-stress balance across each of the samples.

0.75 which indicates the presence of micro-cracking. This conclusion is backed up by the reduced Young's modulus measurements (Figure. 4).

(ii) Anisotropy in the stress state could not reasonably be explained through the thermal mechanism only, as it generates mostly hydrostatic stress. The difference in lateral and axial stress can be attributed to the plastic deformation mechanism. Although quantitative estimations to accurately evaluate this effect are complex, simplified qualitative considerations support the concept of a deformation-induced stress factor. In general, for metal-ceramic composites, two phases are defined by the elastic-plastic metal and the semi-rigid ceramic particles. Uniaxial compression leads to an anisotropic stress state with the ceramic particle phase in compression in the axial direction and in tension for the lateral direction. Thus, combined with the thermally generated tensile stresses, the axial stress for the ceramic phase was expected to have more tensile stresses in the lateral direction and a reduced tensile stress profile for the axial direction. The same trend was observed experimentally at higher volume fractions $f\left(\mathrm{Y}_{2} \mathrm{O}_{3}\right)=0.5$ and 0.75 , though the exact effect was determined through a combination of these competing mechanisms. 
(iii) Stress relaxation through micro-cracking was another mechanism at play. This was supported by the elastic modulus measurements which indicated stress relaxation in the ceramic-rich samples. However, to result in anisotropic stress relaxation, the micro-cracking system is assumed to also be anisotropic with a preferred orientation of the crack plane normal to the axial direction, so the axial stress component is largely relaxed while there is less effect in the lateral direction. This assumption of the anisotropic micro-crack system is also consistent with the anisotropic microstructure observed in Figure. 3(b). The determination of the exact amount and type of micro-cracking is a matter of further study and will be investigated in a future publication.
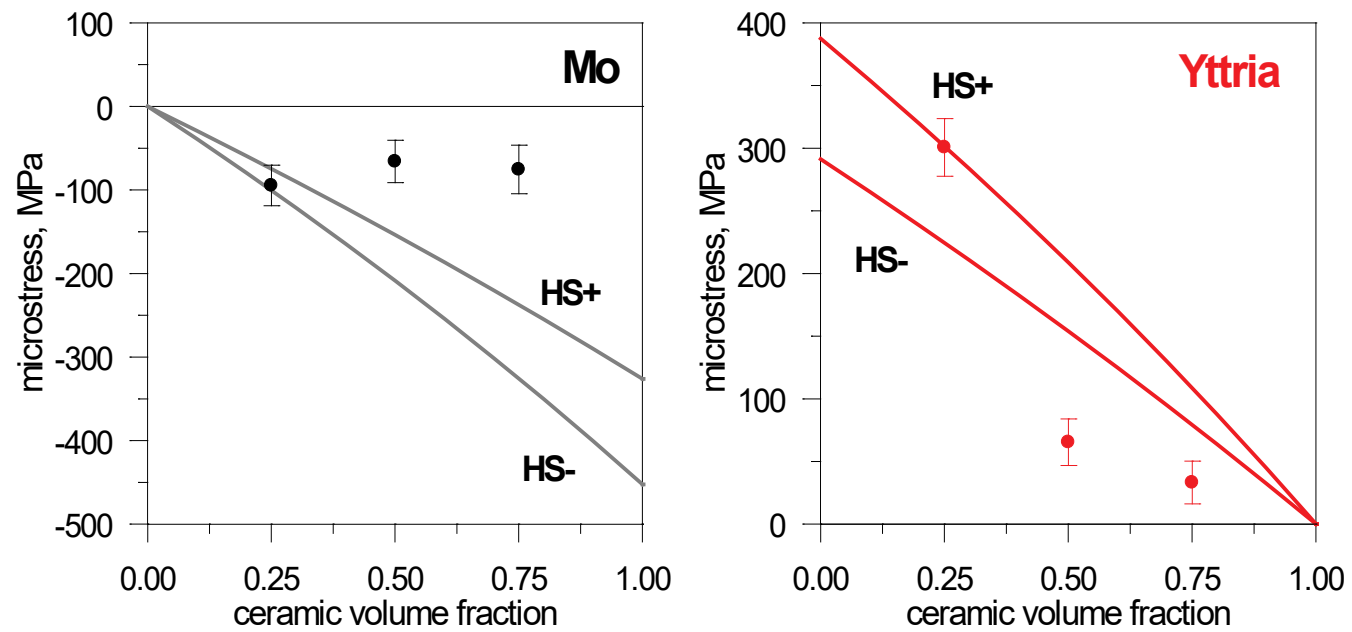

Fig. 6. Micro-stress in each phase due to thermal mismatch: theoretical evaluation vs. experimental data (the axial component was taken as the experimental reference). The Hashin-Shtrikman bounds correspond to $\Delta T=-450^{\circ} \mathrm{C}$.

\section{Conclusion}

This study assessed the residual stresses in a series of metal-ceramic Mo- $\mathrm{Y}_{2} \mathrm{O}_{3}$ FGM analogue samples by means of neutron diffraction. The micro-stresses that formed were predominantly a result of the thermal coefficient mismatch; the micro-stresses were largely isotropic/hydrostatic. The presence of anisotropic stresses highlighted by the difference in the lateral and axial stresses were attributed to plastic deformation, induced in part by the production process. Stress relaxation through the development of micro-cracking was also detected by two independent experimental techniques; these accounted for the reduction in Young's modulus. Overall, the final stress state of the composite samples of various phase compositions was determined experimentally to be a combination of these stress-inducing mechanisms, which are intrinsically linked to the nature of the materials and the technological manufacturing process. Future work will be centred on altering the processing route with the use of cold and hot isostatic pressing, in an attempt to re-evaluate the resultant residual stress by eliminating the directional plastic deformation.

\section{References}

1. Kieback, B., A. Neubrand, and H. Riedel, Processing techniques for functionally graded materials. Materials Science and Engineering: A, 2003. 362(1-2): p. 81-106. http://dx.doi.org/10.1016/S0921-5093(03)00578-1

2. Miyamoto, Y., Functionally Graded Materials: Design, Processing and Applications. 1999: Springer US. http://dx.doi.org/10.1007/978-1-4615-5301-4

3. Chavara, D.T., M. University of Sydney. School of Aerospace, and M. Engineering, Synthesis and Testing of Metal-ceramic Functionally Graded Materials. 2009: University of Sydney. 
4. Ling, Y.-H., et al., Fabrication and evaluation of SiC/Cu functionally graded material used for plasma facing components in a fusion reactor. Journal of Nuclear Materials, 2002. 303(23): p. 188-195. http://dx.doi.org/10.1016/S0022-3115(02)00801-2

5. Nawkaa, S., et al., Synthesis, Characterization and FEM-simulation of $\mathrm{W} / \mathrm{CuCrZr}$ Composites for Extreme Thermal Applications.

6. Australian-Standard, AS 1774.5:2014 Refractories and refractory materials-physical test methods. Method 5: determination of bulk density, apparent porosity and true porosity (ISO 5017:2013, MOD), 2014, Australian Standard.

7. Latella, B.A. and T. Liu, High-Temperature Young's Modulus of Alumina During Sintering. Journal of the American Ceramic Society, 2005. 88(3): p. 773-776. http://dx.doi.org/10.1111/j.1551-2916.2005.00082.x

8. ASTM-International, ASTM E1875-13 Standard test method for dynamic young's modulus, and poisson's ration by sonic resonance, 2013, ASTM International.

9. ASTM-International, ASTM C1259-15 Standard test method for dynamic young's modulus, shear modulus, and poisson's ratio for advanced ceramics by impulse excitation of vibration, 2015, ASTM International.

10. www.matweb.com. [cited 2016].

11. Hashin, Z., The Elastic Moduli of Heterogeneous Materials. ournal of Applied Mechanics, 1962. 29(1): p. 143-150. http://dx.doi.org/10.1115/1.3636446

12. Clyne, T.W. and P.J. Withers, An Introduction to Metal Matrix Composites. 1993: Cambridge University Press. http://dx.doi.org/10.1017/CBO9780511623080 